\title{
Research Progress of Two-dimensional Matrix Detectors in IMRT Dose Verification
}

\author{
Yufen Shang ${ }^{1}$, Yuqing $\mathrm{Xu}^{2}$, Fang Wang ${ }^{3 *}$ \\ ${ }^{1}$ Department of Radiophysics Technology, Dezhou's Second People's Hospital, Dezhou 253000, China \\ ${ }^{2}$ Department of Burns, Dezhou's Second People's Hospital, Dezhou 253000, China \\ ${ }^{3}$ Department of Oncology, Dezhou's Second People's Hospital, Dezhou 253000, China
}

\begin{abstract}
With the continuous development of science and technologies in China, radiotherapy technology in medical field has been very significantly developing, and intensity modulated radiation therapy (IMRT) technology has been the most widely used. This paper first introduces the components and types of two-dimensional matrix detector, two-dimensional ionization chamber matrix detector and two-dimensional semiconductor matrix detector, then analyzes the dosimetric characteristics of the two-dimensional matrix detector. In the end, the various applications of the two-dimensional matrix detector are analyzed and discussed in detail. The paper aims to promote the two-dimensional matrix detector's development in the field of radiotherapy in China.
\end{abstract}

Key words: Two dimensional matrix detector; Intensity modulated radiation therapy technology; Dose verification

Publication date: May, 2021; Publication online: 31 May, 2021

*Corresponding author: Fang Wang, dzeyflzx@163.com

\section{Introduction}

Since entering the new period, radiotherapy technology in China has made remarkable achievements as science and technologies develop. In order to achieve better clinical treatment effects, radiotherapy technology has higher and higher requirements on dose. How to control the accuracy of dose more accurately is the key problem to be solved for current radiotherapy technologies. Intensity-modulated radiation therapy (IMRT) is one of the most advanced radiotherapy technologies over recent years as well as the most widely used radiotherapy technology now, having a great therapeutic effect on the medical clinical oncology treatment $^{[1,2]}$. As old methods of ionization chamber and film gradually outdated, two-dimensional matrix detector has become the most popular IMRT dose verification equipment in China.

\section{The classification of two-dimensional matrix}

\section{detectors}

2.1 Two-dimensional ionization chamber matrix detector

Two-dimensional ionization chamber matrix detector, which is comprised of two-dimensional matrix plate, signal processing circuit, data processing and converter, supports octagonal module and computer data analysis system, and can help measure the point dose and two-dimensional dose distribution online in real time ${ }^{[3]}$. In addition, the two-dimensional ionization chamber matrix is highly efficient in IMRT dose verification, and can also measure the single modulated hadron field and the synthesized radiation field. The two-dimensional plate of the two-dimensional ionization chamber matrix contains hundreds, even thousands of miniature ionization chambers, the sensitive volume of which is small, basically at 0.1 . Compared with the sensitive ionization chamber with large volume, this kind of ionization chamber can reduce the verification errors of the absolute dose of IMRT through modifying and improve the verification accuracy. Currently, the two-dimensional ionization chamber matrix used in clinical tumor radiotherapy is mainly divided into two types that one is the matrix composed of 1020 miniature ionization chambers with $7.62 \mathrm{~mm}$ ionization chamber spacing, and the other is the matrix composed of 729 stereoscopic columnar ionization chambers with $10 \mathrm{~mm}$ ionization chamber spacing. 


\subsection{Two-dimensional semiconductor matrix detector}

The measurement of two-dimensional semiconductor matrix is also a common method for verifying IMRT dose. The components of this detector are consistent with those of two-dimensional ionization chamber matrix detector. Compared with the two-dimensional ionization chamber matrix detector mentioned above, the two-dimensional semiconductor matrix detector has the advantages of high sensitivity and resolution. Besides, the two-dimensional matrix plate in the two- dimensional semiconductor matrix detector contains a semiconductor detector with a sensitive volume of $1.9 \times 10^{-5} \mathrm{~cm}^{3}$. Like the two-dimensional ionization chamber matrix detector, the semiconductor matrix detector is divided into two types. One is a semiconductor detector, containing a matrix plate in 484 measurement area, that is separated by $7.07 \mathrm{~mm}$ in turn in the central part of the matrix plate, and a matrix, having 445 semiconductor detectors with thickness of $60 \mu \mathrm{m}$, that is separated by $14.14 \mathrm{~mm}$ from those in the peripheral part in the central part. The other is a two-dimensional semiconductor matrix detector with a measured area of 832 $\mathrm{cm}^{2}$ and 1527 detectors with a space distance of $7.07 \mathrm{~mm}$ in the matrix, and such a space design can ensure a higher resolution of matrix measurement and improve the accuracy of IMRT dose verification.

We can infer that the effective measurement area interval of two-dimensional matrix detector is $484-832 \mathrm{~cm}^{2}$ via comparing and summarizing two kinds of twodimensional matrix detectors. In addition, this condition must be taken into account in the validation of dose to ensure the validation efficiency and accuracy. And if the space distance between detectors in the two-dimensional matrix plate is different, the measurement resolution of the matrix will be deviated. To correct the temperature and pressure value in the measurement process, the two-dimensional ionization chamber matrix plate needs to be equipped with temperature and pressure sensors, but the two-dimensional semiconductor matrix is not applicable. There is another significant difference between 2-D ionization chamber matrix detector and 2-D semiconductor matrix detector because of different structures and materials, so their effective sensitive volume is far from each other. The sensitivity of semiconductor detector is obviously much higher than that of ionization chamber detector. In terms of dose verification, the semiconductor detector has a range of 330 cGy, and it can maintain a good dose-measuring characteristic after long working hours. Therefore, all the circumstances that need to be considered should be integrated to select the most suitable two-dimensional matrix detector in the process of dose verification of IMRT.

\section{Dosimetric properties of a two-dimensional}

\section{matrix detector}

\subsection{Linearity of dose response and measurement repeatability}

The dosimetric quality of the second-order matrix detector should be guaranteed in the process of dose verification, while the dosimetric response linearity is one of the most important dosimetric indexes of the two-dimensional matrix detector. Jersenecker and Nelms et al. found that the linear deviation of two-dimensional semiconductor matrix detector was $<1 \%$ when the verification range was $5-300 \mathrm{cGy}$, and the linear deviation of two-dimensional ionization chamber matrix detector was $<0.2 \%$ when the verification range was $100-500 \mathrm{cGy}$. Besides the linearity of the dose response, the measurement repeatability is also an important index. Moreover, Spezi et al. considered that the repeatability of experimental data was about $0.2 \%$ in a few hours under the condition of a fixed validation range for $100 \mathrm{cGy}$ and a beam of $225 \mathrm{~cm}^{2}$, and the repeatability increased over time, eventually remaining around $1 \%$. In addition, Amerio put the two dimensional ionization chamber matrix under the $6 \mathrm{MV}$ X-ray to study the dose linear, which demonstrated that the measurement repeatability of ionization chamber matrix was very good under these conditions. Therefore, it can be obtained that the dose response linearity and measurement repeatability of the two-dimensional matrix can meet the dosimetric requirements for IMRT dose verification.

\subsection{Energy dependence and lateral scattering}

Owing to current imperfections of detector technology and the characteristics of manufacturing materials, two-dimensional matrix detector in the process of production does not guarantee that every dose response micro probes are exactly the same, so the two-dimensional matrix to the detectors for Jane occurs before leaving the factory, in order to achieve the consistency and accuracy of dose measurement. To explore the dose-dependent of two dimensional ionization chamber matrix, American experts in radiotherapy technology put the ionization chamber under the accelerator photon energy of 6,10 and $15 \mathrm{MV}$ to get relevant numerical readings. After comparing with the dosimeter reading, it was found that the energy response 
error of the ionization chamber was $<1.2 \%$, which was a relatively ideal number, proving that the energy dependence of the ionization chamber was small. Part of the Chinese scholars had the corresponding experiments on two-dimensional ion chamber under different beam area of dose distribution, which displayed that there was a significant difference for the measured value between the existential center detector and ionization chamber due to the existence of the lateral scattering center. Based on this, the scholars conducted a convolution correction and found that the correction matrix measurement accuracy was improved obviously after correction. Therefore, before the application of two-dimensional matrix, the measurement of energy dependence under different energy photon lines and the calibration of lateral scattering should be carried out for the micro detector in order to reduce the measurement deviation.

\subsection{Directional response}

When conducting directional response detection on two-dimensional semiconductor matrix detector and two-dimensional ionization chamber matrix detector, researchers found that there was a certain degree of directional response difference in both of them. The two-dimensional matrix detector is composed of many miniature detectors arranged in a certain layout. Moreover, the incident angle of the ray under the same field is not exactly in unison, so when the central axis of the field is not perpendicular to the plane of the matrix detector, especially when the ray is parallel to the plane of the detector, the possible difference in directional responsiveness must be taken into account. Over recent years, with the further study of two-dimensional matrix detectors in China, it was found that if the two-dimensional matrix is added to the periphery of the appropriate thickness of the module, then this structure can reflect a good IMRT dose verification effect. The researches have shown that if the two-dimensional matrix plate is properly moved in the actual operation, the difference in directional response can be weakened or even eliminated. Therefore, the directional response should be adjusted when the two-dimensional matrix detector is used to verify the dose of IMRT.

\section{The application of two-dimensional matrix}

\section{detector}

\subsection{Application of ionization chamber matrix}

Two-dimensional ionization chamber matrix detector has excellent performance in IMRT dose verification.
Meanwhile, as a verification tool, it is also simple, efficient, accurate and reliable in the process of use. Due to its high verification accuracy, many radiotherapy experts have made many innovative applications of two-dimensional ionization chamber matrix. In the process of studying the ionization chamber matrix, Spezi used less than a quarter of an hour to assemble and debug the ionization chamber matrix, and then found a lot of meaningful properties of which in the dosimetry test. Firstly, the ionization chamber matrix had an independent energy response, which was a new breakthrough for the verification of IMRT's small field dose. Secondly, Spezi also found that the dose verification curve of the ionization chamber matrix was consistent with the scanning curve of the ionization chamber in the tank under IMRT. Moreover, Jia et al. found that the final results were similar after measuring different ionization chamber matrices. Besides, Ren et al. verified 100 cases of IMRT, and found that the verification results basically met the international dose deviation standard, and the pass rate of the entire IMRT dose could reached up to $97 \%$. When Wizluk used the ionization chamber matrix to measure the flatness and repeatability of the field, he found that the measurement accuracy of the two-dimensional ionization chamber matrix could achieve good accelerator quality control. Cybernetan discovered that the ionization chamber matrix could not only provide good quality control for the accelerator, but also improve the efficiency of IMRT dose verification in the research center of two-dimensional matrix.

\subsection{Applications of semiconductor matrices}

Compared with the traditional film dosimeter, two-dimensional semiconductor matrix dosimetry characteristics, the dose of linearity and repeatability characteristics in particular is more outstanding. As it was specifically designed to verify the IMRT doses of a validation work, the actual operation of which is more simple than film dosimeter, its measuring precision stays at a good level, in the measurement of absolute dose also has the very good application. Chen et al. tested the accuracy of dose verification of two-dimensional semiconductor matrix through experiments, and the test results showed that when the dose position was limited under standard conditions, the pass rate of relative doses of all radiation fields could reach $85 \%-100 \%$. Many radiotherapy institutions are using two-dimensional semiconductor matrix to verify the dose of IMRT, and in the process of clinical application, they have also innovated matrix verification methods to replace the 
combination of film dosing method and ionization chamber matrix for IMRT dose verification method.

\section{Conclusion}

In summary, how to control the accuracy of dose more accurately is of great significance for the improvement of current radiotherapy technology level. Two-dimensional ionization chamber matrix detector has great advantages in IMRT dose verification and can significantly improve the efficiency of IMRT dose verification and has a very outstanding contribution in the offset of semiconductor matrix, and exerts important effects in the field of radiation therapy in China.

\section{References}

[1] Faghihi Moghaddam F, Bakhshandeh M, Ghorbani M, Mofid B. 2020. Assessing the out-of-field dose calculation accuracy by eclipse treatment planning system in sliding window IMRT of prostate cancer patients. Comput Biol Med 127: 104052.

[2] Patni A, Rastogi M, Gandhi AK, Rath S, Sr. SSN, Khurana R, et al. 2020. Clinico-Dosimetric Correlation Of Doses To Dysphagia Aspiration Related Structures (DARS) With Acute Toxicities In Post-Operative Patients Of Oral Cavity Carcinoma Treated With Dysphagia Optimized Versus Standard IMRT. International Journal of Radiation Oncology*Biology*Physics 108.

[3] Pigorsch SU, Kampfer S, Oechsner M, Mayinger MC, Mozes P, Devecka M, et al. 2020. Report on planning comparison of VMAT, IMRT and helical tomotherapy for the ESCALOX-trial pre-study. Radiat Oncol 15: 253.

[4] Li LF, Zhai HZ, Wang YZ, Ming X, Han BL. Research on directional response of a two-dimensional ionization chamber matrix detector based on intensity-modulated radiotherapy[J]. International Journal of Biomedical Engineering,2020,43(04):297-303.

[5] Duan XJ, Dai HY, Zhao LR, Zhou YB. Application analysis of a new home-made two-dimensional matrix in the dose verification of intense-modulated radiotherapy for thoracic and abdominal tumor[J]. Chinese Medical Equipment Journal,2020,41(07):64-68.

[6] Wang XC, Wang SY, Huo XQ, Zhang QQ, Hong ZY, Lu P, Wu P, Liu B, Wang YT, Miao ZH. Effect and correction of thermoplastic film fixed plate on the pass rate of three dimensional dose verification of IMRT program $[\mathrm{J}]$.Chinese Journal of Medical Physics,2020,37(03):273-276.

[7] Yang CY, Zhou YY, Cao XJ, Du X, Luo SM, Xue X, He ZJ, Wang J. Study on the verification method of target volume, dose of organs at risk and 2-D dose distribution in 8 sets of accelerator intensity modulated radiotherapy in Jiangsu province[J].Chinese Journal of Radiological Medicine and Protection,2020(02):129-130-131-132-133.

[8] Zhao Q, Wu XY, Chang XB, Feng T, Yang D, Zhang K, Qu XM, Wang XM, Deng J. The feasibility study on verification of Acuros BV algorithm for calculating relative dose distribution by two-dimensional matrix ionization chamber[J].Chinese Journal of Radiological Medicine and Protection,2020(01):59-60-61-62-63.

[9] Shi L. Application analysis of two dimensional ionization chamber matrix in accelerator virtual wedge measurement $[\mathrm{J}]$. World Latest Medicine Information, 2019,19(41):119-120.

[10] Liu W. Research on methods for increasing the SNR of RBOTDA system[D]. North China Electric Power University, 2018. 